\title{
Dipole Antenna for Interstitial Microwave Heating in a Dissipative medium: A Design Prospective
}

\author{
A. S. Farahat ${ }^{*}$, E. M. El-Dewany", F. M. El-Hefnawi", Y. M. Kadah ${ }^{* *}$, A. A. Youssef* \\ "Microwave Engineering Department, Electronics Research Institute, Asmaa@eri.sci.org \\ "Department of Biomedical Engineering, Cairo University
}

\begin{abstract}
Interstitial antennas are useful for producing localized power as in the hyperthermia treatment of tumors. Simple analytical forms for the generated electric field, the radiated power, and the input impedance are used in this paper to study the characteristics of a short dipole in a dissipative medium similar to the white matter of the brain tissues for a frequency range from 0.2 to $2 \mathrm{GHz}$. These analytical forms are found to be a fast efficient technique for designing a small dipole antenna that can be used to produce a concentrated power around the antenna that can be used to produce localized regional heating for hyperthermia treatment of brain tumors. It is found that a dipole whose length and radius are nearly $7 \mathrm{~mm}$ and $0.3 \mathrm{~mm}$ respectively operating at nearly $400 \mathrm{MHz}$ is quite suitable to produce the required localized power. Also FDTD method is used to verify the validity of these analytical forms.
\end{abstract}

\section{I- Introduction}

Most of researches on microwave antennas for medical applications have focused on producing hyperthermia for medical treatments and monitoring various physiological parameters. One of the main objectives of using microwaves in hyperthermia for cancer treatment is to develop the capability of delivering therapeutic heat to deep-seated tumors without over heating the surrounding healthy tissues [1]. This requires the radiated power from the antenna to be dissipated and localized in small area. When interstitial antennas are used in conjunction with other types of treatment, such as brachytherapy, the existing surgical tracks can be used to insert interstitial antennas in the body [2]. Antennas applied to elevate the temperature of cancer tissues can be located inside or outside of the patient's body. The types of the used antennas depend on the location. For instance, waveguide or low-profile antennas are more suitable to be externally positioned. On the other hand, monopole or dipole antennas fed by a coaxial cable can be designed for internal use [3]. Among the large varieties of antennas that may be used in this application, including waveguide applicators, phased array applicators, and other applicators of the capacitive or inductive type [4], the use of interstitial antennas has recently been of great interest [5].

To design an interstitial antenna to be used for microwave heating of brain tumors, different parameters such as the length and radius of the antenna, operating frequency, and impedance matching properties have to be adjusted, in order to get a fast decaying electric field, and an amount of localized radiated power around the antenna, which is a required property for tumors hyperthermia. The design procedure requires identifying and quantifying the trade-offs between these various design parameters.

This paper presents a parametric study to show the effects of the dipole length, radius and operating frequency on the generated electric field, the dissipated power, and the input impedance of such dipole embedded in a dissipative medium similar to the white matter of the brain tissues. We have also studied various tissues of the brain, like the gray matter and the cerebellum. It is found that they have similar performance like the white matter. However, due to the space limit, the results here are mainly based on the characteristics of the white matter. This parametric study is based on simple analytical forms. The basic theory of this analysis is discussed in details through the following section. Section III presents the results and discussions. Finally. 


\section{The $23^{\text {rd }}$ National Radio Science Conference (NRSC 2006) \\ March 14-16, 2006}

Section IV presents a comparison between the results obtained by the analytical techniques and the corresponding results obtained numerically by using finite-difference time-domain (FDTD) [6].

\section{II- Theory}

In hyperthermia applications, the main interest is primarily in the field quite close to the antenna where most of the heating takes place. Thus, the near electric field of a dipole is much more involved than the far field. On the other hand implanting an antenna inside the patient is an invasive process. Thus, it is required to minimize the total size of the implanted antenna. Hence, this study is mainly focused on the analysis of a short dipole antenna such that $\beta h<1$ and $\alpha h<1$ where $\alpha$ and $\beta$ are the attenuation and phase constants of the wave in the dissipative medium respectively and $h$ is the dipole half length. This section presents the basic equations which are required to design a short dipole for hyperthermia applications.

The total electric field of a short dipole located along the $z$-axis including the near and far field components is given by [7]

$$
E_{\theta}(r)=\frac{j \omega \mu I_{z}(0) h}{4 \pi k}\left(\frac{k}{r}-\frac{j}{r^{2}}-\frac{1}{k r^{3}}\right) e^{-j k r} \sin \theta,
$$

where $\omega$ is the operating angular frequency, $\mu$ is the permeability of the medium, $I_{z}(0)$ is the input current ,$k=\beta-j \alpha$ is the complex propagation constant of the medium and $r$ is distance from the center of the dipole to the observation point, Figure(1). The above equation can be used to calculate the electric field due to a short dipole at any observation point whose $r$ is greater than the dipole half length $h$.

The localized power inside a near-field sphere surrounding such short dipole is the difference between the power supplied to the antenna and power transferred outside this sphere. This localized power can be obtained as [7]

$$
P=\frac{\left|I_{z}(0)\right|^{2}}{2}\left(R_{0}-\frac{\beta^{2} h^{2} \zeta_{e} \psi_{d r}}{6 \pi(\Omega-3)}\left(1+\frac{\alpha}{\beta} L\right) e^{-2 \alpha r}\right)
$$

where, $R_{0}$ is the input resistance of the dipole,

$$
L=\frac{2}{\left(1+\alpha^{2} / \beta^{2}\right) \beta r}+\frac{4 \alpha / \beta}{\left(1+\alpha^{2} / \beta^{2}\right)^{2} \beta^{2} r^{2}}+\frac{2}{\left(1+\alpha^{2} / \beta^{2}\right)^{2} \beta^{3} r^{3}},
$$

$\psi_{d r}=2 \log \frac{h}{a}-2$,

$\Omega=2 \log \frac{2 h}{a}$,

$$
\zeta_{e}=\frac{\varpi \mu}{\beta}
$$

and $a$ is the radius of the dipole. 
For design purpose, it is required also to obtain the input impedance of such short dipole where the maximum power transfer can be obtained by designing an appropriate matching network. The input impedance of such short dipole can be obtained as follows [7]:

$$
Z=\frac{1}{G_{0}+j B_{0}},
$$

where

$$
\begin{gathered}
G_{0}=\frac{2 \pi}{\zeta_{e} \Psi_{d r}}\left\{\frac{2 \alpha}{\beta}\left[\beta h+\frac{2 \beta^{3} h^{3} F}{3}\left(1-\frac{\alpha^{2}}{\beta^{2}}\right)\right]+\frac{\beta^{4} h^{4}}{3(\Omega-3)}\left(1-10 \frac{\alpha^{2}}{\beta^{2}}+5 \frac{\alpha^{4}}{\beta^{4}}\right)\right\} \\
B_{0}=\frac{2 \pi}{\zeta_{e} \Psi_{d r}}\left\{\begin{array}{r}
\beta h\left(1-\frac{\alpha^{2}}{\beta^{2}}\right)+\frac{\beta^{3} h^{3} F}{3}\left(1-6 \frac{\alpha^{2}}{\beta^{2}}+\frac{\alpha^{4}}{\beta^{4}}\right) \\
-\frac{\beta^{4} h^{4}}{3(\Omega-3)} \frac{\alpha}{\beta}\left(5-10 \frac{\alpha^{2}}{\beta^{2}}+\frac{\alpha^{4}}{\beta^{4}}\right)
\end{array}\right\}
\end{gathered}
$$

and

$$
F=1+\frac{3 \log (2)-1}{\Omega-3}
$$

By using the above three equations, one can obtain the electric field distribution, the dissipated power in a closed volume and the input impedance of a short dipole embedded inside a dissipative medium.

\section{III- Results and Discussion}

In this section the previously mentioned theory presented in Sec.(2) is used to study the characteristics of a short dipole embedded inside a dissipative medium similar to the white matter of the brain tissues. The electrical properties of such dissipative medium for different frequencies are listed in Table (I) [8]. It can be noted that for such parameters the propagation constant $\beta$ and the attenuation coefficient $\alpha$ at lower frequencies are nearly of the same order of magnitude. For higher frequencies the propagation constant $\beta$ gets much larger than the attenuation coefficient $\alpha$. This means that at low frequencies the proposed medium is nearly a conducting medium, while at high frequencies it would be more like an insulating medium.

Figure (2) shows the electric field distribution as a function of the radial distance between the dipole and the observation point along the normal direction to the axis of the dipole. In this case the short dipole is assumed to be of a half length $h=3.5 \mathrm{~mm}$ and a radius $a=0.3 \mathrm{~mm}$. The minimum wavelength in the present simulation including the effect of the surrounding medium is nearly $25 \mathrm{~mm}$. Thus, the maximum half length of this dipole in terms of the operating wavelength is nearly 0.14 which can be considered as a short dipole. It can be noticed for such dipole that the decay rate of the electric field in the near field region to the dipole is much higher at lower frequencies than the corresponding one at high frequencies. However, at larger distances the decay rate of lower frequencies becomes much slower than the corresponding one of high frequencies.

This behavior can be explained by considering the different terms of equation (1). At low frequencies, where $k$ is small, the near field region of small $r$ is more dominated by the algebraic terms $r^{-n}$ rather than the exponential term $e^{-\alpha r}$. Thus, the dominant behavior of the near field at low frequencies is dominated by $1 / r^{3}$ which represents a very high decay rate. On the other hand, at high frequencies, the wave number $k$ has 


\section{The $23^{\text {rd }}$ National Radio Science Conference (NRSC 2006) \\ March 14-16, 2006}

Faculty of Electronic Engineering, Menoufiya University, Egypt.

large values. Hence, the mechanism of the field decay has a different scenario. In the very close region to the dipole the dominant decay is dominated by the term $1 / r^{3}$. Then the decay rate becomes more dominated by the terms $1 / r^{2}$ and $1 / r$ at larger distances from the antenna. As the spacing between the antenna and the observation point increases, the effect of the algebraic terms $r^{-n}$ becomes weaker compared with the effect of the exponential attenuation term $e^{-\alpha r}$ which becomes the dominant decay rate.

Table I

Electrical properties and the corresponding propagation constants of the white matter inside the brain tissues .

\begin{tabular}{|c|c|c|c|c|c|}
\hline Frequency $(\mathrm{GHz})$ & ${ } \varepsilon$ & $\sigma$ & $\alpha$ & $\beta$ & $|k=\beta-j \alpha|$ \\
\hline 0.2 & 47 & 0.37 & 9.8 & 30.8 & 42.1 \\
\hline 0.4 & 42 & 0.44 & 12 & 55.7 & 57.2 \\
\hline 0.8 & 39 & 0.56 & 16.6 & 106 & 107.3 \\
\hline 1.0 & 38.5 & 0.62 & 18.6 & 131 & 132.0 \\
\hline 1.5 & 37 & 0.79 & 24.2 & 192 & 193.5 \\
\hline 2.0 & 36 & 1.0 & 31.1 & 253 & 254.7 \\
\hline
\end{tabular}

Figure (3) shows the localized power inside a near-field sphere surrounding such short dipole of a radius $r>h$ at the same frequencies. It can be noted that at low frequencies, more power is concentrated around the dipole. By increasing the excitation frequency this localized power around the dipole penetrates to a larger distances. This can also be explained in the same manner as the electric field decay.

For hyperthermia application, the antenna is required to deliver most of its input power to the surrounding medium. Thus, the input impedance has to be matched to the feeding line which is usually designed to be $50 \Omega$. The input impedance of such short antenna at the present dissipative medium can be calculated directly by using equation (3). Figure (4) shows the frequency dependence of the input impedance for short dipoles of different lengths. It can be noticed that the best matching with respect to the $50 \Omega$ is nearly $400 \mathrm{MHz}$ where the real part of the input impedance is nearly the same as the transmission line. The remaining capacitive part of the input impedance can be cancelled by adding an inductive load. Figure (5) shows the input impedance dependence on the dipole half lengths for different frequencies. It is found that the suitable half-length is nearly $3 \mathrm{~mm}$ excited by $400 \mathrm{MHz}$.

Finally, the input impedance dependence on the dipole radius is investigated for different frequencies, Figure (6), it is found that for low frequencies, specifically near $400 \mathrm{MHz}$, the suitable radius for best matching is nearly $0.3 \mathrm{~mm}$.

\section{IV- Verification by FDTD}

In this section, finite difference time domain (FDTD) [6] is used to verify the validity of the analytical technique discussed in Sec. II. The dimensions of the dipole are the same as those in Fig. 2 and the operating frequency is assumed to be $400 \mathrm{MHz}$. The excitation voltage is assumed to be $7.4 \mathrm{~V}$. This excitation voltage is chosen such that the amplitude of the input current is nearly $0.3 \mathrm{~A}$ to be the same as the corresponding one in Fig.2. This dipole is embedded in a homogeneous medium similar to the white matter of the brain, with a dielectric constant $\varepsilon_{r}=42$ and $\sigma=0.44$.

Figure (7) shows a comparison between the calculated electric field distribution by using the analytical technique and the corresponding result by using FDTD. It can be noticed the good agreement between the two techniques. The input impedance obtained by using FDTD in this case is found to be $Z=59.583-j 143.69$. The corresponding result obtained by using the analytical technique is $Z=54.261-\mathrm{j} 112.77$ which is quite close to the result of the FDTD. 


\section{V-Conclusion}

This paper presents a simple analytical technique to design an interstitial antenna for hyperthermia treatment of tumors. This analytical technique is verified by comparison with FDTD. From results and discussions it can be concluded that low frequencies between $200 \mathrm{MHz}$ to $400 \mathrm{MHz}$ are the optimum choice for implanted antenna in a dissipative medium like the brain. A dipole characteristics with $\mathrm{h}=3.5 \mathrm{~mm}$ and $\mathrm{a}=0.3 \mathrm{~mm}$ excited by 400 $\mathrm{MHz}$ leads to a localized power and better impedance matching properties. The proposed antenna would be useful for different types of brain tumors as it has almost the same performance in the various brain tissues.

\section{REFERENCES}

[1] P. C. Cherry, and M. F. Iskander, "Calculations of heating patterns of an array of microwave interstitial antennas", IEEE Trans. Biomedical Eng., vol. 40, No. 8, March 1993 pp. 1934-1943

[2] C. T. Coughlin, E. B. Douple, J. W. Strohbehn, W. L. Eaton, B. S. Trembly, and T. Z. Wong, "Interstitial hyperthermia in combination with brachytherapy," Radiology, vol. 148, pp. 285-288, July 1983.

[3] J. Kim and Y. R. Samii, "Implanted antennas inside a human body: simulations, designs and characterizations," IEEE Trans. Microwave Theory Tech., vol. 52, No. 8, pp. 1934-1943, Aug 2004.

[4] M. Hiraoka, M. Mitsumori, N. Hiroi, S. Ohno, Y. Tanaka, Y. Kotsuka, and K. Sugimachi, "Development of RF and microwave heating equipment and clinical applications to cancer treatment in Japan," IEEE Trans. Microwave Theory Tech., vol. 48 , No. 11, pp. 1789-1799, Nov 2000.

[5] A. M. Tumeh and M. F. Iskander, "Performance comparison of available interstitial antennas for microwave hyperthermia," IEEE Trans. Microwave Theory Tech., vol. 37, No. 7, pp. 1126-1133, July 1989.

[6] A. Taflove, S. C. Hagness, Computational Electrodynamics: the finite difference time domain method ( ${ }^{\text {nd }}$ edition), Artech House, Boston, 2000

[7] R. P. King and C. W. Harraison, Antennas and Waves: A modern approach, The MIT press, 1969

[8] http://www.fcc.gov/fcc-bin/dielec.sh 


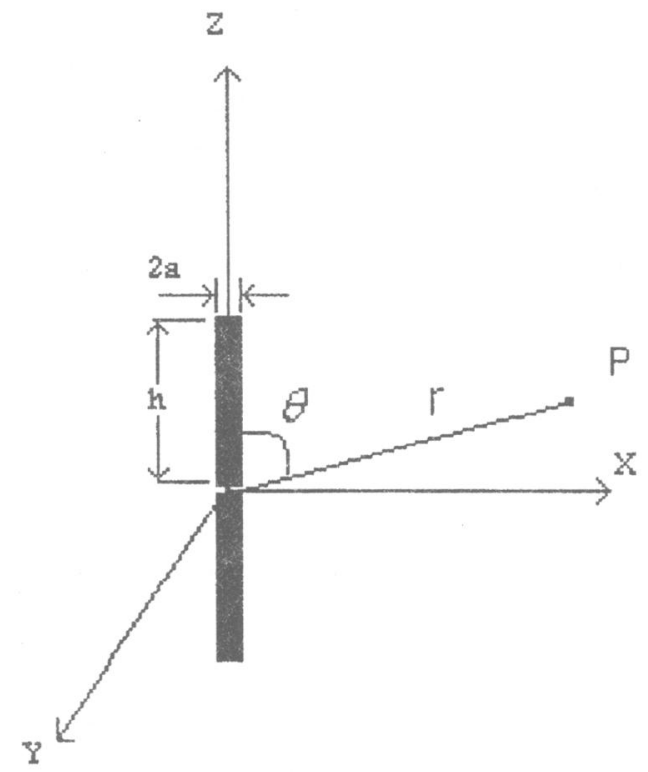

Fig.1 Dipole configuration

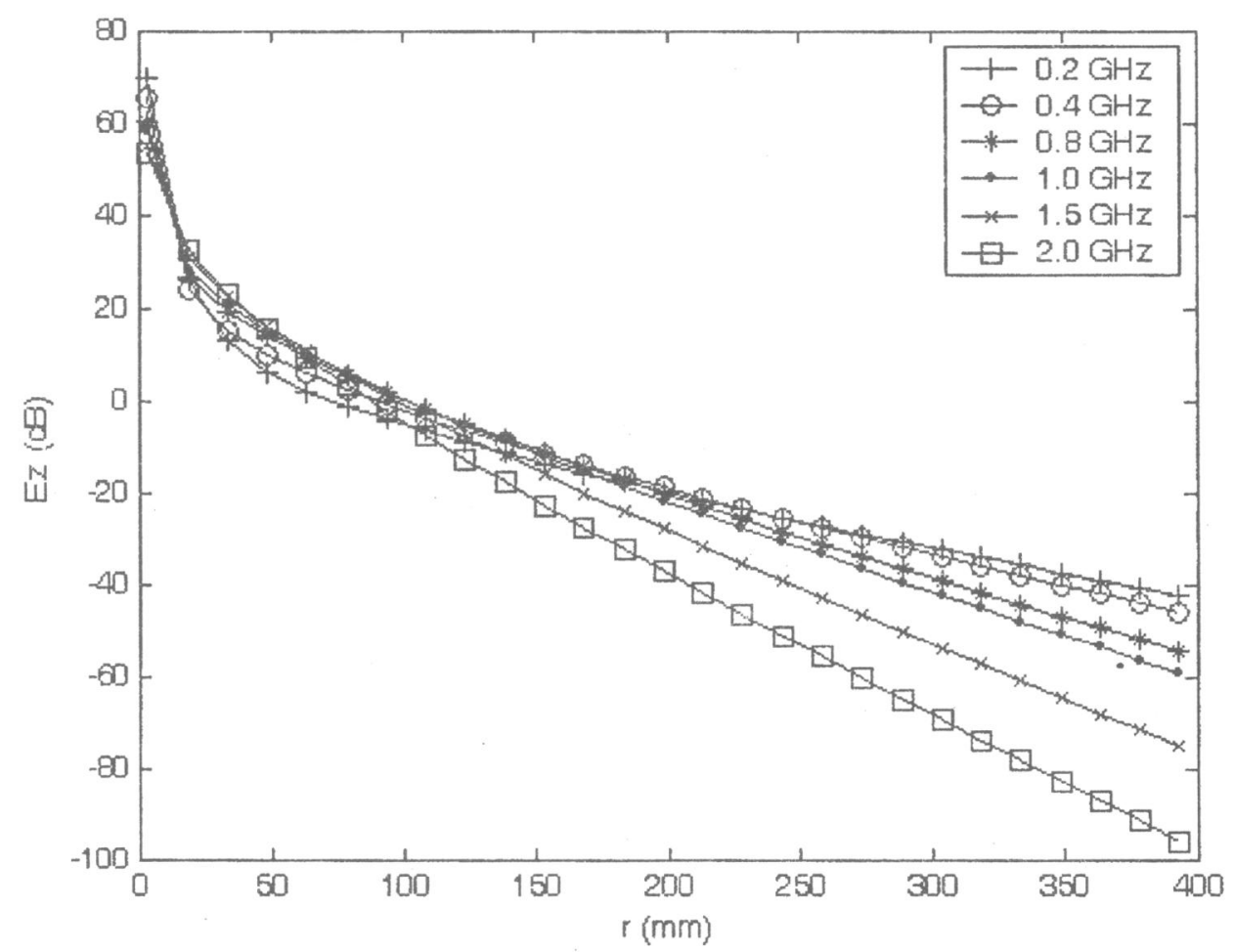

Fig. 2-a Frequency dependence of electric field intensity extension along the $x$-axis $(h=3.5 \mathrm{~mm}, a=0.3 \mathrm{~mm}$, $\left.\mathrm{I}_{2}(0)=0.3 \mathrm{~A}\right)$ 
The $23^{\text {rd }}$ National Radio Science Conference (NRSC 2006)

March 14-16, 2006

Faculty of Electronic Engineering, Menoufiya University, Egypt.

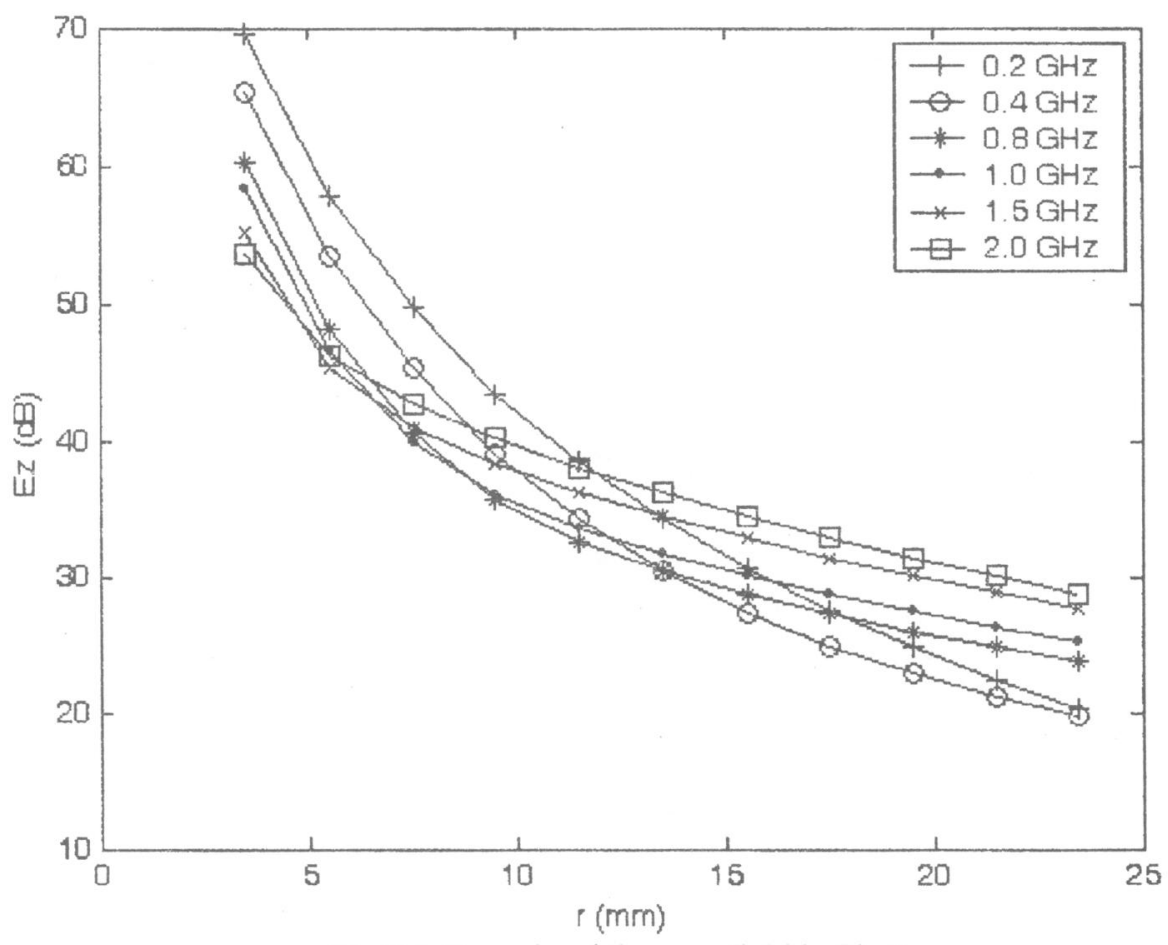

Fig.2-b Zoom in of the near field in Fig.2-a.

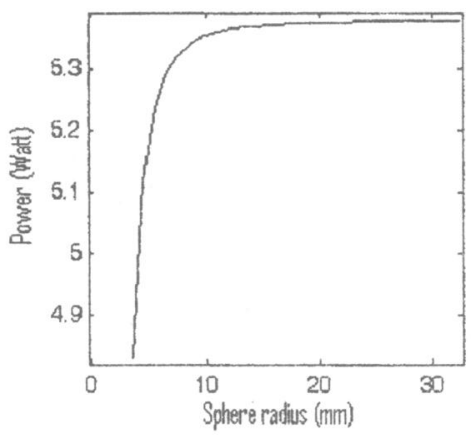

(a) $f=0.2 \mathrm{GHz}$

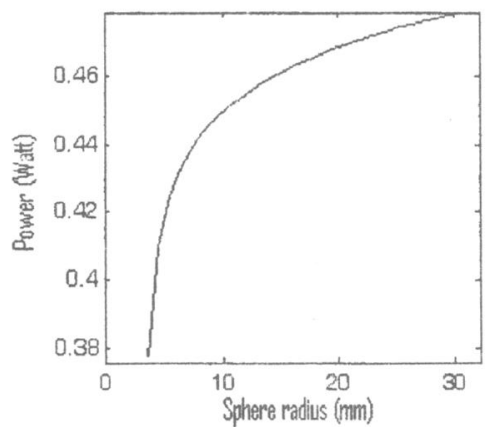

(c) $f=1.5 \mathrm{GHz}$

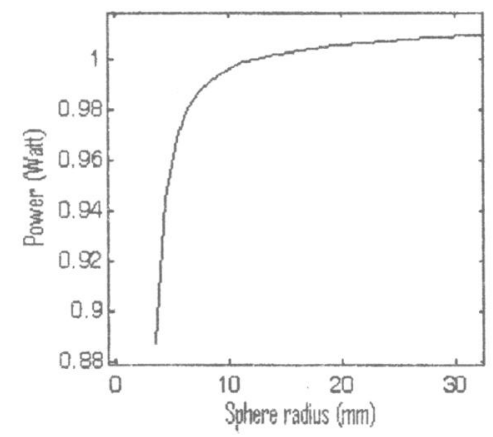

(b) $f=0.8 \mathrm{GHz}$

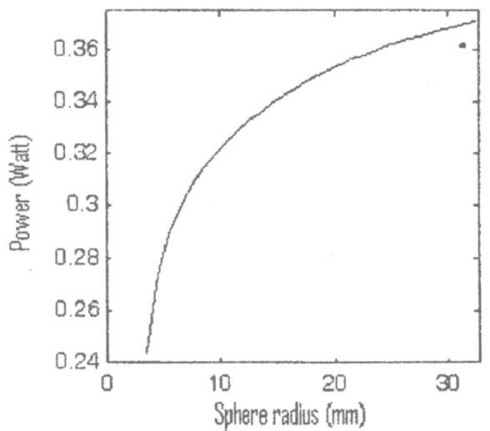

(d) $f=2 \mathrm{GHz}$

Fig.3 Power contained within a sphere of radius $r>h$ surrounding the dipole for different frequencies and constant input current $\mathrm{I}_{z}(0)=0.3 \mathrm{~A}(\mathrm{~h}=3.5 \mathrm{~mm}, \mathrm{a}=0.3 \mathrm{~mm}$, ) 
The $23^{\text {rd }}$ National Radio Science Conference (NRSC 2006)

March 14-16, 2006

Faculty of Electronic Engineering, Menoufiya University, Egypt.

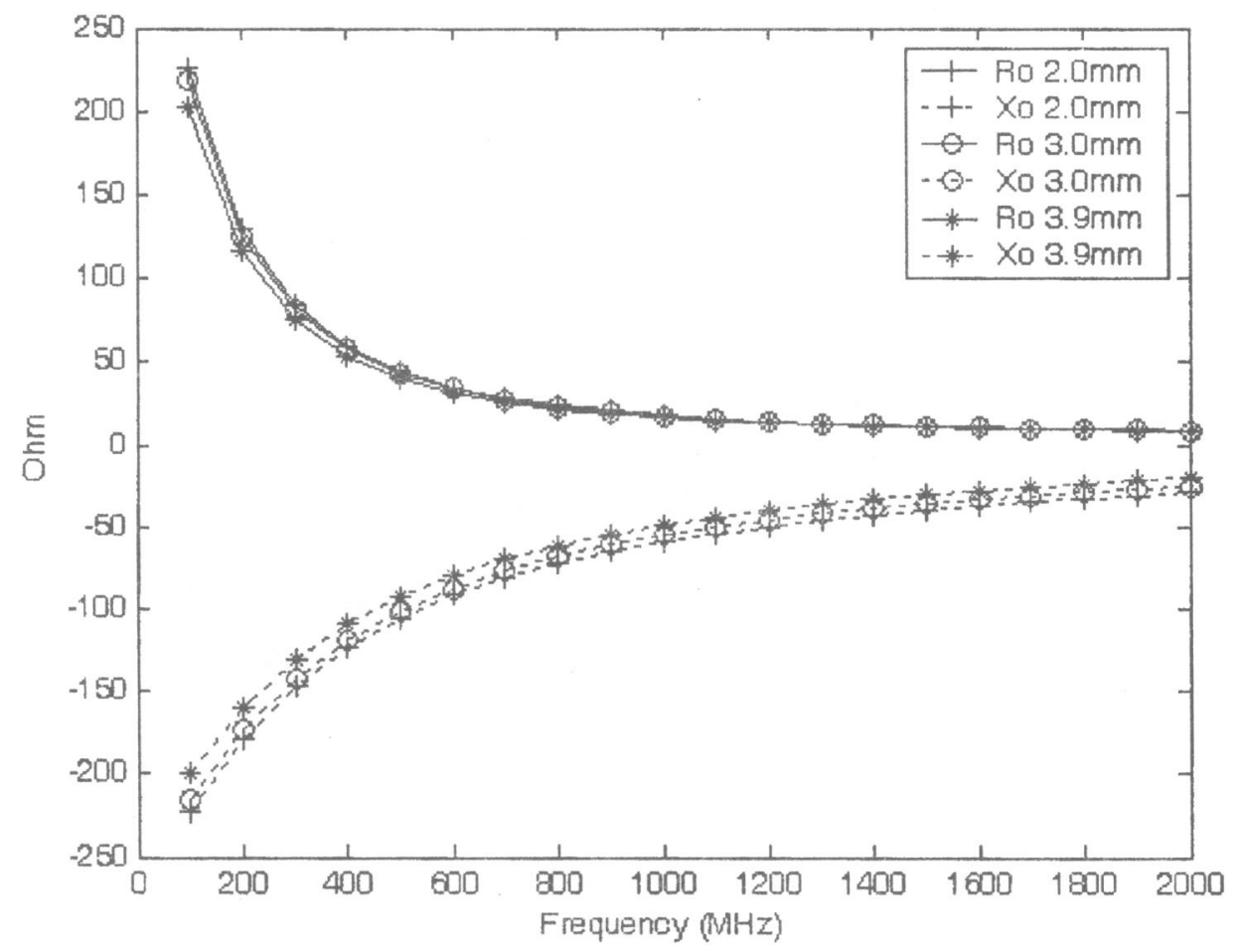

Fig.4 Frequency dependence of the input impedance for short dipoles of different lengths embedded in the white mater $(a=0.3 \mathrm{~mm})$.

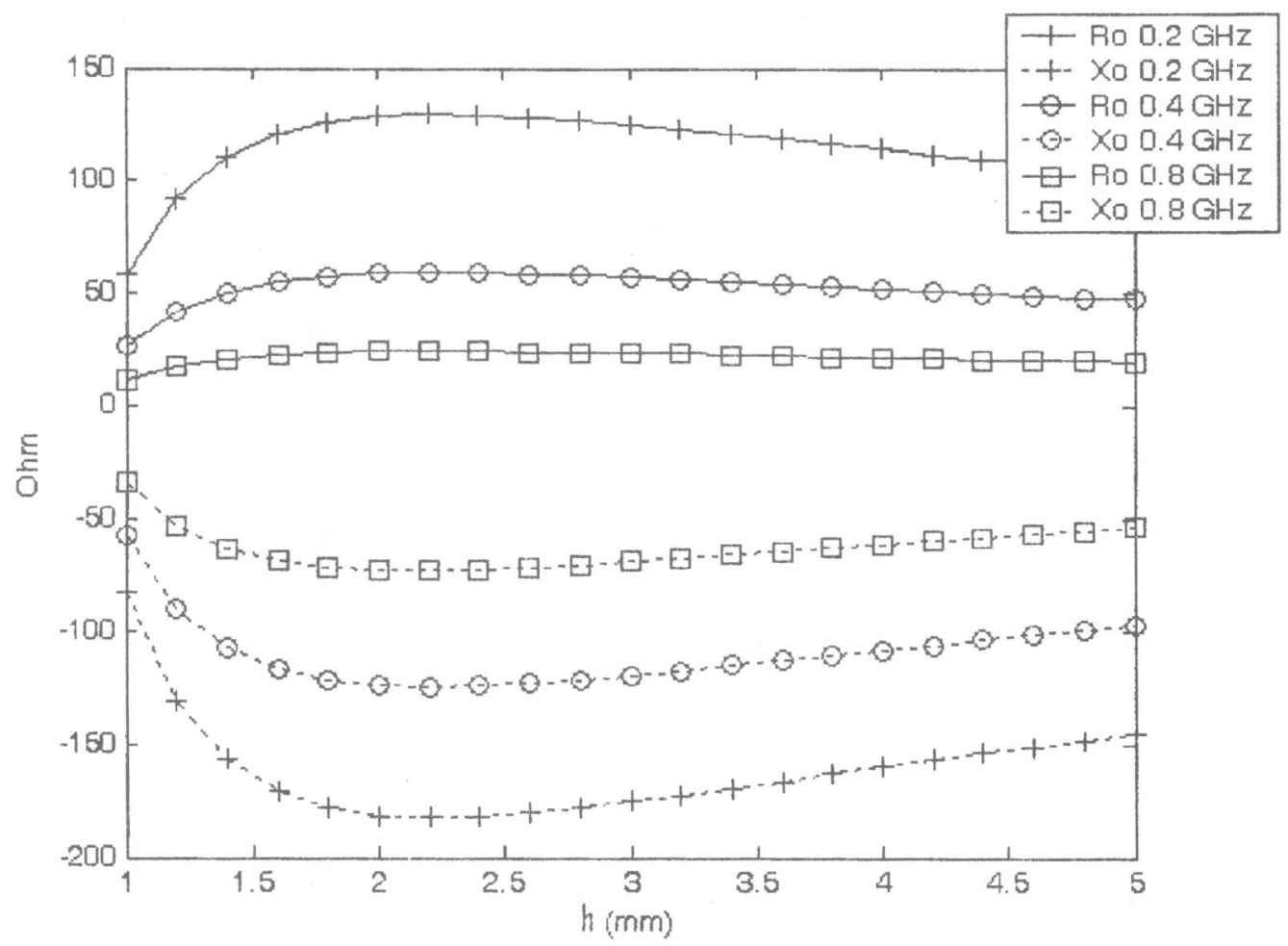

Fig.5 Dipole impedance dependence on the dipole half-length in the white mater $(a=0.3 \mathrm{~mm})$ 
The $23^{\text {rd }}$ National Radio Science Conference (NRSC 2006)

March 14-16, 2006

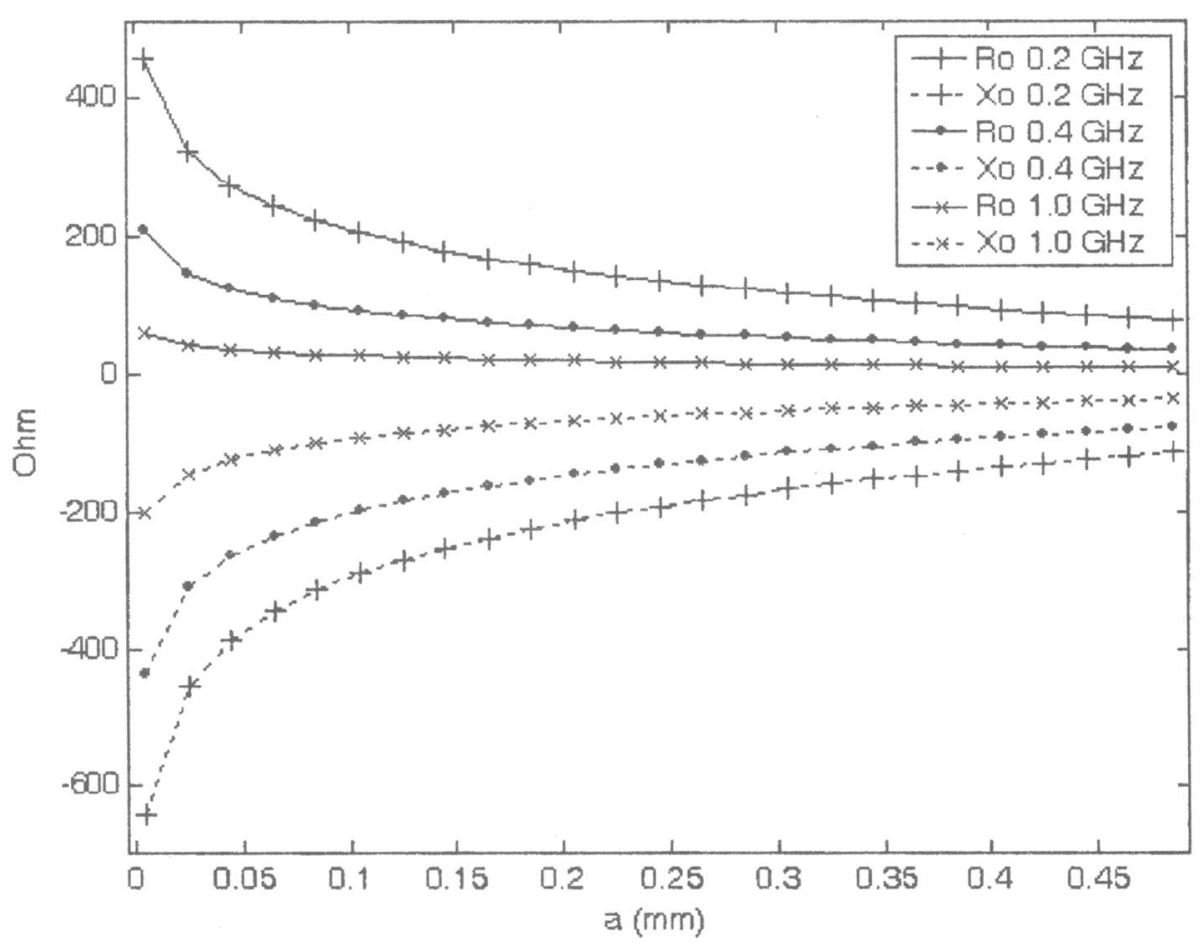

Fig.6 radius dependence of the input impedance for short dipoles embedded in the white mater $(h=3.5 \mathrm{~mm})$.

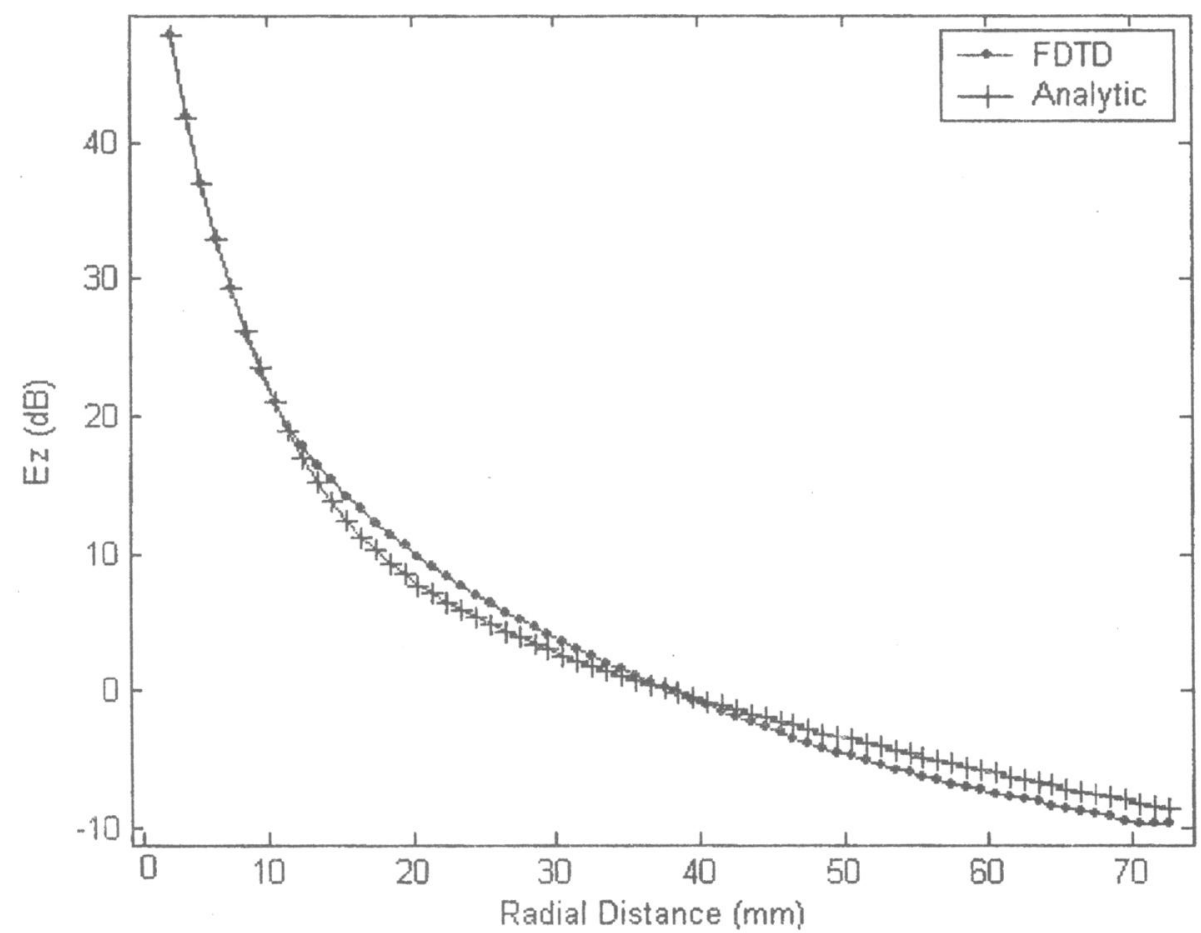

Fig. 7 Frequency dependence of electric field intensity extension along the $x$-axis $(h=3.5 \mathrm{~mm}, a=0.3 \mathrm{~mm}, V=7.4 \mathrm{~V}$, Frequency $=400 \mathrm{MHz}$ ) 